\title{
Sistematização da Assistência de Enfermagem Perioperatória em cirurgia da cavidade oral ambulatorial
}

\author{
Perioperative Nursing Care System for ambulatory surgery of the oral cavity \\ Enfermería de Atención Perioperatoria para cirugía ambulatorial de la cavidad oral
}

Adriana Santana de Vasconcelos ${ }^{1}$, Evanísia Assis Góes Araújo² ${ }^{2}$ Valquiria Farias Bezerra Barbosa ${ }^{3}$, Laureana de Vasconcelos Sobral ${ }^{4}$, Francisca Márcia Pereira Linhares ${ }^{5}$

RESUMO: Objetivo: descrever a construção e a validação de protocolo de Sistematização da Assistência de Enfermagem Perioperatória (SAEP), aplicado à cirurgia oral menor no Centro de Especialidades Odontológicas, vinculado à uma instituição de ensino superior em Pernambuco. Método: relato de experiência no qual foram utilizados os Padrões Funcionais de Saúde de Gordon para coleta de dados, identificação dos Diagnósticos de Enfermagem segundo a Taxonomia NANDA - I e posterior validação com pacientes atendidos entre 2008 e 2009. Conclusão: O protocolo de SAEP proposto permitiu que o processo de Enfermagem na formação em nível de graduação não fosse apenas abordado sob o ponto de vista teórico. O relato dos estudantes extensionistas evidenciou a viabilidade e a eficácia da SAEP, além de suas vantagens sobre a qualidade da assistência e do processo de trabalho da Enfermagem.

PALAVRAS-CHAVE: Enfermagem. Assistência Perioperatória. Boca.

ABSTRACT: Objective: to describe the construction and validation of the Perioperative Nursing Care System (PNCS) protocol applied to minor oral surgeries at the Center for Dental Technology, "Faculdade ASCES", Caruaru, Pernambuco state. Method: an experience report in which the Gordon Functional Health Patterns were used for data collection, with identification of Nursing Diagnoses according to the diagnostic terminology by NANDA - I, and subsequent validation with patients treated between 2008 and 2009 . Conclusion: the PNCS protocol proposed allowed the process of nursing training at undergraduate level to be approached beyond the theoretical point of view. The report of extension students showed the effectiveness of the Perioperative Nursing Care System on the quality of care and nursing work process.

KEYwords: Nursing. Perioperative Care. Mouth.

RESUMEN: Objetivo: describir la construcción y validación del protocolo de Atención de Enfermería Perioperatoria Sistematizada (AEPS), aplicado a la cirugía oral menor en el Centro de Especialidades Odontológicas, vinculado a la institución de enseñanza superior en Pernambuco. Método: relato de experiencia en el que se utilizaron los Padrones Funcionales de Salud de Gordon para la recogida de datos, identificación de los Diagnósticos de Enfermería según la Taxonomía NANDA - l y su posterior validación con pacientes atendidos entre los años 2008 y 2009. Conclusión: el protocolo de la AEPS propuesto permitió que el proceso de Enfermería, en la formación a nivel de graduación, no sólo fuese abordado desde el punto de vista teórico. El relato de los estudiantes extensionistas demostró la viabilidad y eficacia de la AEPS, además de sus ventajas sobre la calidad de la atención y sobre el proceso de trabajo de la Enfermería.

PALABRAS CLAVE: Enfermería. Atención Perioperatoria. Boca.

${ }^{1}$ Mestre em Enfermagem em Promoção da Saúde. Universidade de Pernambuco e Universidade Estadual da Paraíba - UPE/UEPB. Professora. Faculdade ASCES. Av. Venezuela, 229, Bairro Universitário. CEP 55016-470. Caruaru, PE, Brasil.Telefone: (81) 3724-5535. E-mail: drikasv@ig.com.br

${ }^{2}$ Mestre em Saúde Pública. Universidade Federal da Paraíba. Professora. Faculdade ASCES. Caruaru, PE, Brasil. E-mail: evanisia@terra.com.br

${ }^{3}$ Professora. Universidade Federal de Pernambuco. Recife, PE, Brasil. E-mail: valquiriaenfermeira@ yahoo.com.br

${ }^{4}$ Enfermeira Responsável Técnica. Centro de Especialidades Odontológicas (CEO) vinculado à Faculdade ASCES. E-mail: laureanasobral@ asces.edu.br

${ }^{5}$ Doutoranda. Programa Interdisciplinar em Ciências Humanas. Universidade Federal de Santa Catarina modalidade DINTER. Mestre em Bioquímica. Universidade Federal de

Pernambuco. Professora. Instituto Federal de Educação Ciência e Tecnologia de Pernambuco. Campus Pesqueira. Pesqueira, PE, Brasil. E-mail: marciapl27@ gmail.com 


\section{Introdução}

A cirurgia ambulatorial ganhou importância na década de 1960, nos Estados Unidos, pelo desenvolvimento de técnicas e substâncias anestésicas que permitiram a recuperação mais rápida e a alta precoce do paciente. Atualmente, é uma alternativa que possibilita a redução dos riscos de infecção operatória e, consequentemente, dos custos financeiros ${ }^{1}$.

Embora o tempo de permanência do cliente no serviço de cirurgia seja reduzido, isto não exclui a necessidade de uma assistência multiprofissional e de Enfermagem de qualidade. O Enfermeiro qualificado para o cuidado perioperatório é capaz de desenvolver técnicas que possibilitem uma assistência de alta qualidade com o menor custo, mediante uma metodologia própria, a Sistematização da Assistência de Enfermagem Perioperatória -SAEP, objetivando a segurança do paciente e da equipe envolvida no ato operatório².

No Brasil, a discussão acerca do acesso à Odontologia especializada no sistema público é recente ${ }^{3,4}$, porém relevante, à medida que a Política Nacional de Saúde Bucal lançada em 2004 viabiliza a oferta da atenção secundária através de Centros de Especialidades Odontológicas - $\mathrm{CEOs}^{5,6}$. Os CEOs são estabelecimentos especializados em diagnóstico do câncer bucal, Periodontia, Cirurgia Oral Menor, Endodontia e atendimento a pessoas com necessidades especiais ${ }^{4,7}$.

Entre os procedimentos cirúrgicos mais realizados nos CEOs, estão aqueles destinados à prevenção e ao tratamento do câncer bucal, e a exodontia de elementos inclusos, não erupcionados no período habitual. Esta ocorrência é considerada uma alteração no desenvolvimento do elemento dentário ${ }^{8}$. As duas condições sugerem a realização de procedimentos cirúrgicos ambulatoriais.

Há uma lacuna na produção científica no contexto das cirurgias odontológicas ambulatoriais, relacionada à forma como se realiza a assistência de Enfermagem. Talvez esta lacuna ocorra em decorrência do Enfermeiro não estar envolvido diretamente na assistência à cirurgia ambulatorial odontológica. As autoras foram motivadas a desenvolver este trabalho pela necessidade, enquanto docentes de uma instituição privada de educação superior, de buscar um campo de práticas clínicas em que o estudante de Enfermagem pudesse vivenciar a assistência perioperatória em caráter ambulatorial. Ao mesmo tempo, o CEO que funcionava como campo de práticas para os estudantes de Odontologia da mesma IES necessitava implantar o serviço de Enfermagem no centro cirúrgico, tendo em vista a ampliação do aporte de procedimentos cirúrgicos de pequena e média complexidade. Foi então proposto um projeto de extensão que, juntamente com atividades de ensino e pesquisa, pudesse integrar assistência odontológica e de Enfermagem na produção de um novo conhecimento. Dessa maneira, buscou-se particularizar ações gerais da assistência de Enfermagem cirúrgica à cirurgia odontológica de caráter ambulatorial.

A SAEP possibilita à Enfermagem atuar de forma organizada, sistematizada e humanizada. É uma atividade privativa do Enfermeiro, de acordo com a Lei do Exercício Profissional de Enfermagem n ${ }^{\circ} 7498 / 86^{9}$. Objetiva levantar e analisar as necessidades individuais do paciente a ser submetido ao procedimento anestésico-cirúrgico; minimizar riscos decorrentes da utilização dos materiais e equipamentos necessários ao desenvolvimento do procedimento; prever, prover e gerenciar os recursos humanos, e implementar a assistência de Enfermagem de forma integral, individualizada, documentada, participativa e avaliada, focando o paciente nos períodos de pré, trans e pós-operatório ${ }^{10,11}$.

Destaca-se, na SAEP, dentre outras ações, a educação para o autocuidado e o apoio emocional a pacientes e familiares acerca dos problemas de saúde relacionados à intervenção cirúrgica, permitindo a interação entre o Enfermeiro e o paciente e, principalmente, a redução da ansiedade e do medo pré-operatórios ${ }^{12,13}$. Esse enfoque reforça, sobretudo, a relevância do estudo da SAEP para a eficácia na qualidade da assistência de Enfermagem prestada ao paciente submetido à cirurgia oral menor ${ }^{14}$.

Apesar de um número significativo de Enfermeiros concordar com a necessidade de sistematizar o atendimento nos cenários de atuação profissional, a adesão ao método ainda é pequena devido às dificuldades encontradas para sua implementação, entre as quais se pode exemplificar: sobrecarga de funções, insuficiência quantitativa de profissionais, instrumentos gerenciais e assistenciais inadequados, necessidade de atualização dos conhecimentos científicos e reduzida autonomia da Enfermagem ${ }^{15,16}$.

Mesmo diante das dificuldades vivenciadas pelos Enfermeiros, diversos estudos descrevem que os roteiros e protocolos destinados à consulta de Enfermagem auxiliam na coleta de dados relevantes, reduzindo o tempo destinado ao registro da assistência prestada; possibilitam, ainda, correlacionar diagnósticos e prescrições de Enfermagem, norteando a assistência que será prestada aos sujeitos ${ }^{14,17,18}$.

Ante ao exposto, este trabalho destina-se a descrever a experiência das autoras na construção e na validação de um protocolo de Sistematização da Assistência de Enfermagem Perioperatória aplicado a cirurgias ambulatoriais da cavidade oral.

\section{Método}

Trata-se de um estudo descritivo, do tipo relato de experiência, sobre a construção de um protocolo de SAEP aplicado à cirurgia oral menor e a implantação do Serviço de Enfermagem no CEO, vinculado à Faculdade Associação Caruaruense de Ensino Superior, situada em Caruaru, Pernambuco, entre os anos de 2008 e 2011.

A elaboração do protocolo de SAEP para pacientes submetidos à cirurgia da cavidade oral obedeceu às seguintes etapas:

1) Elaboração de uma proposta de protocolo com base em revisão da literatura. Para a coleta de dados, foram utilizados como referência os Padrões Funcionais de Saúde de Gordon ${ }^{19}$, 
devido à necessidade de coletar dados relevantes que auxiliem na identificação dos Diagnósticos de Enfermagem (DE). A terminologia diagnóstica adotada foi a North American Nursing Diagnosis Association International (NANDA - I) ${ }^{20}$, uma vez que já existe ampla literatura relacionada a esta taxonomia voltada para a Enfermagem perioperatória, abrangendo os DE mais frequentemente encontrados nos sujeitos submetidos à experiência cirúrgica. Para a construção das intervenções de Enfermagem, realizou-se a revisão da literatura sobre os cuidados de Enfermagem dispensados ao cliente que se submete à cirurgia oral. Assim, foram utilizados inicialmente 13 padrões funcionais de saúde e dez DE;

2) A coleta de dados para validação do protocolo foi desenvolvida por um grupo de três professores, um Enfermeiro e 15 estudantes extensionistas durante os anos de 2008 e 2009, período no qual foram feitas as adequações necessárias ao instrumento, mediante reuniões científicas mensais, sendo, posteriormente, encaminhado para um revisor especialista em sistematização da assistência de Enfermagem, para realizar análise e validação do instrumento em epígrafe.

O Projeto de Extensão "Sistematização da Assistência de Enfermagem Perioperatória (SAEP) para Cirurgia da Cavidade Oral" foi avaliado e aprovado pelo comitê de ética em 18 de julho de 2008.

Durante a primeira consulta Odontológica e de Enfermagem, os pacientes eram orientados e aqueles que concordaram assinaram o Termo de Consentimento Livre e Esclarecido, que já consta do prontuário do CEO, conforme preceitua a Resolução n. ${ }^{\circ}$ 196/96 CNS. Tendo em vista ser um centro odontológico-cirúrgico escola, a atividade de extensão é aprovada pelo Comitê de Ética e Pesquisa da Faculdade ASCES desde 17 de Julho de 2008.

\section{Resultados e discussão}

\section{Proposta inicial do protocolo}

A proposta inicial de um protocolo para pacientes submetidos à cirurgia oral menor surgiu durante a elaboração do Trabalho de Conclusão do Curso de Aperfeiçoamento em Sistematização da Assistência de Enfermagem, oferecido pela Faculdade ASCES, que visava a instrumentalizar professores para o ensino da SAE, de forma contextualizada, no Curso de Graduação em Enfermagem.

Nesta instituição, já funcionava o CEO e nele estava inserido o serviço de cirurgia menor ambulatorial da cavidade oral. No entanto, os pacientes atendidos por este serviço não recebiam assistência por parte de profissionais Enfermeiros. As professoras visualizaram a oportunidade de estabelecer um campo de prática no contexto da própria IES para os estudantes do curso de Enfermagem e a possibilidade de agregar qualidade à assistência já oferecida pelos Odontólogos da IES. Foi implantado então um projeto de extensão denominado 'Projeto de Extensão Integrado Odontologia-Enfermagem'. Paralelamente ao desenvolvimento das atividades extensionistas, durante o ano de 2008, foi implantado o serviço de Enfermagem no $\mathrm{CEO}$, mediante a contratação de um profissional Enfermeiro para assumir a responsabilidade técnica pela assistência de Enfermagem, tendo em vista a demanda crescente por procedimentos cirúrgicos de pequena e média complexidades.

O Centro Cirúrgico é uma unidade fechada, na qual são realizados procedimentos anestésico-cirúrgicos. A complexidade da unidade requer que o Enfermeiro possua conhecimento científico, responsabilidade, habilidade técnica, estabilidade emocional e a compreensão das relações humanas, a fim de que a dinâmica de trabalho do serviço de Enfermagem, aliada ao relacionamento entre os profissionais que atuam na referida unidade, possa acontecer de forma harmoniosa, visando a segurança e bem-estar do paciente. A qualidade da assistência de Enfermagem prestada, tanto no período que antecede a cirurgia como após a realização da mesma, interfere nos resultados do procedimento realizado ${ }^{21}$.

A partir desta motivação, foi construído um protocolo para a realização da consulta de Enfermagem baseado nos padrões funcionais de saúde de Gordon e na Taxonomia NANDA ${ }^{19,20}$. Inicialmente, o protocolo foi constituído por todos os padrões propostos por Gordon, a saber: percepção e controle de saúde; padrão nutricional-metabólico; eliminações; cognitivoperceptivo; autopercepção e autoconceito; desempenho de papel e relacionamento; padrão sexual-reprodutivo, resposta e tolerância ao estresse; crença e valor; atividade e exercício, e padrão de sono e repouso ${ }^{19}$. O protocolo também possuía um conjunto de DE sugeridos a partir da revisão da literatura que trata da SAEP. Os diagnósticos utilizados foram: integridade tissular prejudicada, risco de infecção, conhecimento deficiente, risco de broncoaspiração, medo e ansiedade. A partir destes diagnósticos, foi elaborado um conjunto de nove intervenções de Enfermagem associadas.

\section{A validação do instrumento}

O protocolo foi construído por professoras com experiência assistencial superior a cinco anos na área de Enfermagem Médico-Cirúrgica. Após sua elaboração, passou pela correção de Professora Mestre, com experiência comprovada na área de $\mathrm{SAE}$, além da contribuição do Enfermeiro responsável técnico pelo serviço de Enfermagem do CEO. Após serem realizadas as devidas correções, o protocolo foi utilizado na realização da consulta de Enfermagem, respeitando a Resolução n. ${ }^{\circ}$ 358/2009 do Conselho Federal de Enfermagem, no âmbito do projeto de extensão com concentração na assistência perioperatória em cirurgia odontológica ambulatorial ${ }^{22}$.

$\mathrm{O}$ referido protocolo foi aplicado em pacientes adultos e idosos submetidos à cirurgia oral. Os pacientes que foram incluídos no estudo, entre os anos de 2008 e 2009, tiveram as seguintes indicações cirúrgicas: exérese dos elementos inclusos, cirurgia pré-protética e retirada de tumores. Após este período de utilização, foi possível perceber a necessidade de adequação do protocolo, especialmente com relação à 
fase de coleta de dados, para melhor esclarecer o processo de assistência realizado no intra e no pós-operatório.

Tal necessidade ocorreu devido às peculiaridades da cirurgia oral e foram apontadas pelos 15 extensionistas, enquanto desenvolviam suas atividades assistenciais. Desta forma, descrevem-se, a seguir, as modificações realizadas no instrumento. Os padrões: eliminações; autopercepção e autoconceito; desempenho de papel e relacionamento; valor e crença; atividade e exercício, e sono e repouso foram suprimidos do instrumento, pois poucos pacientes atendidos apresentavam alterações destes padrões. Estes detalhes foram percebidos no momento da realização da consulta de Enfermagem no pré-operatório mediato (momento em que a equipe decide que o paciente terá uma abordagem cirúrgica), na consulta do pré-operatório imediato (neste caso de cirurgia ambulatorial, uma a duas horas antes da realização do procedimento) e durante o acompanhamento, em unidade de recuperação pós-anestésica.

Vivenciar estas fases da SAEP junto ao paciente cirúrgicoodontológico permitiu ao grupo envolvido detectar as demandas particulares desta clientela e adequar o protocolo de assistência a estas necessidades. Foi introduzido um campo para registro da assistência de Enfermagem transoperatória. A assistência intraoperatória é o período correspondente à intervenção sobre o sítio cirúrgico. O pós-operatório foi dividido em imediato, que se caracteriza pela alta do paciente da sala de recuperação pós-anestésica (RPA) até 48 horas do pós-operatório, e proservação, que corresponde ao pós-operatório tardio, compreendendo as 48 horas do pósoperatório até a alta do paciente ${ }^{23}$. Foram descritos os DE mais frequentes no período de recuperação pós-anestésica e suas intervenções relacionadas. Estas alterações foram testadas até a obtenção do modelo que se utiliza atualmente (Apêndice).

\section{A aplicabilidade do protocolo}

A nova versão do protocolo foi diagramada a partir do programa Microsoft Office Standard 2003, com a licença Open License: 41101817. A escolha por esse formato de diagramação se deveu ao fato de que o impresso deveria ser de fácil manuseio para as equipes envolvidas e baixo custo de impressão, além de permitir a continuidade das fases da SAEP todas as vezes que o paciente viesse ao serviço para finalização do tratamento. Os procedimentos cirúrgicoodontológicos muitas vezes precisam ser realizados em mais de um elemento dentário para sua conclusão; desta forma, o protocolo contribui para que não haja necessidade de repetição de informações. Isto promove a agilidade na realização da consulta do pré-operatório mediato, imediato, trans e pós-operatório, especialmente porque as fases da SAEP, com frequência, ocorrem mais de uma vez.

A realização da SAEP conduzida através do protocolo em foco permite a superação da problemática de que o processo de Enfermagem na formação em nível de graduação seja apenas abordado sob o ponto de vista teórico ${ }^{24}$. A superioridade desta abordagem foi constatada mediante o relato dos extensionistas sobre as atividades assistenciais vivenciadas no Centro Cirúrgico do CEO. Os estudantes afirmam que conseguiram constatar a viabilidade da SAEP, bem como suas vantagens sobre a qualidade da assistência e do processo de trabalho da Enfermagem.

Dessa maneira, as autoras avaliam que a aplicação de um protocolo de SAEP permite ao estudante de Enfermagem reconhecer a importância da aplicação do processo de Enfermagem e que esta experiência poderá contribuir para a repetição da assistência sistematizada em suas futuras vivências profissionais.

\section{Considerações finais}

A construção de um protocolo de sistematização da assistência de Enfermagem perioperatória aplicada a cirurgias da cavidade oral mostrou-se um desafio para o grupo de autoras em decorrência da escassez de referências em torno da temática. Desta forma, considera-se que a construção deste protocolo constitui-se em uma tecnologia inovadora para a Enfermagem.

Por outro lado, o curso de Aperfeiçoamento em Sistematização da Assistência de Enfermagem para os professores Enfermeiros do Curso de Graduação em Enfermagem da Faculdade ASCES constituiu-se em um agente facilitador da construção deste protocolo assistencial. Esta atualização possibilitou a aproximação dos preceitos teóricos e práticos da assistência de Enfermagem, os quais, muitas vezes, permanecem dicotômicos na prática profissional do Enfermeiro. Pode-se observar o importante papel que as IES possuem em superar a dicotomia teoria-prática pela utilização de práticas pedagógicas que possibilitem a contextualização dos conhecimentos nos cenários de atuação profissional.

Desta forma, ressalta-se a importância de relatar a construção de estratégias que norteiem a assistência de Enfermagem sistematizada, especialmente em áreas pouco exploradas, como a cirurgia oral realizada ambulatorialmente. A divulgação das experiências auxilia no compartilhamento de conhecimentos para a construção da assistência de Enfermagem baseada em evidências ${ }^{25}$. $\mathrm{O}$ aprimoramento da prática científica da Enfermagem contribui para a qualidade da assistência e para o reconhecimento social da Enfermagem, estimulando estudantes, professores e demais profissionais de Enfermagem a refletir e reconstruir o perfil profissional da Enfermagem brasileira.

\section{Referências}

1. Flório MCS, Galvão CM. Cirurgia Ambulatorial: identificação dos diagnósticos de enfermagem no período perioperatório. Rev Latino-Am Enferm. 2003 Set-Out;11(5):630-7. 
2. Smeltzer SC, Bare BG. Brunner e Suddarth. Tratado de Enfermagem Médico - Cirúrgica. 10. ed. Rio de Janeiro: Guanabara koogan; 2006.

3. Ferreira CA, Loureiro CA. Custos para implantação e operação de serviço de saúde bucal na perspectiva do serviço e da sociedade. Cad Saúde Pública. 2008;24:2071-80. PMid:18813683. http:// dx.doi.org/10.1590/S0102-311X2008000900013

4. Figueiredo N, Goes PSA. Construção da atenção secundária em saúde bucal: um estudo sobre os Centros de Especialidades Odontológicas em Pernambuco, Brasil. Cad Saúde Pública. 2009;25:259-67. http://dx.doi.org/10.1590/S0102-311X2009000200004

5. Frazão P, Narvai PC. Saúde bucal no Sistema Único de Saúde: 20 anos de lutas por uma política pública. Saude Debate. 2009;33(81):64-71.

6. Pucca Junior GA, Costa JF, Chagas LD, Silvestre RM. Oral health policies in Brazil. Braz Oral Res. 2009;23(Suppl 1):9-16. http:// dx.doi.org/10.1590/S1806-83242009000500003

7. Chaves SCL, Barros SG, Cruz DN, Figueiredo ACL, Moura BLA, Cangussu MCT. Política Nacional de Saúde Bucal: fatores associados à integralidade do cuidado. Rev Saúde Pública. 2010;44(6). http://dx.doi.org/10.1590/S0034-89102010005000041

8. Nogueira AS. Considerações gerais sobre dentes inclusos. In: Nogueira AS. Abordagem contemporânea dos dentes inclusos -o diagnóstico ao tratamento cirúrgico ortodôntico. São Paulo: Santos; 2004. cap. 1, p. 3-10.

9. Conselho Federal de Enfermagem. Código de ética dos profissionais de enfermagem. Lei $n^{\circ} 7.498$ de 25/06/1986. Rio de Janeiro: COFEN; 2010.

10. Possari JF. Sistematização da Assistência de Enfermagem Perioperatória. In: Possari JF. Centro Cirúrgico: planejamento, organização e gestão. São Paulo: Iátria; 2004.

11. Sociedade Brasileira de Enfermeiros de Centro Cirúrgico, Recuperação Anestésica e Centro de Material e Esterilização - SOBECC. Práticas recomendadas. 5. ed. São Paulo: SOBECC; 2009

12. Piccoli M, Galvão CM. Enfermagem perioperatória: identificação do diagnóstico de enfermagem risco para infecção fundamentada no modelo conceitual de Levine. Rev LatinoAm Enferm. 2001;9(4):37-43. http://dx.doi.org/10.1590/ S0104-11692001000400007
13. Matos FGOA, Píccoli M, Schneider JF. Reflexões sobre aspectos emocionais do paciente cirúrgico. Cienc Cuid Saúde. 2004 Jan-Abr;3(1):93-8.

14. Saragioto I, Tramontini C. Sistematização da assistência de enfermagem perioperatória: estratégias utilizadas por enfermeiros para sua aplicação. Cienc Cuid Saude. 2009 jul./set.;8(3):366-37.

15. Campos SMCL. Sistemática da assistência da enfermagem perioperatória: percepção de enfermeiros assistenciais. Rev SOBECC. 2000;5(4):21-5.

16. Sperandio DJ, Évora YDM. Planejamento da assistência de enfermagem: proposta de um software-protótipo. Ver LatinoAm Enferm. 2005;13(6):937-43. http://dx.doi.org/10.1590/ S0104-11692005000600004

17. Wagner LR, Thofehrn MB, Amestoy SC, Porto AR, Arrieira ICO. Relações interpessoais no trabalho: percepção de técnicos e auxiliares de enfermagem. Cogitare Enferm. 2009;14(1):107-13.

18. Amante LN, Rossetto AP, Schneider DG. Sistematização da ssistência de enfermagem em unidade de terapia intensiva sustentada pela Teoria de Wanda Horta. Rev Esc Enferm USP. 2009;43(1):54-64. PMid:19437854. http://dx.doi.org/10.1590/ S0080-62342009000100007

19. Carpenito - Moyet LJ. Diagnósticos de Enfermagem - Aplicação à Prática Clínica. 10. ed. Porto Alegre: Artmed; 2005.

20. North American Nursing Diagnosis Association. Diagnósticos de enfermagem da NANDA: definições e classificação: 2007-2008. Porto Alegre: Artmed; 2008

21. Stumm EMF, Maçalai RT, Kirschner RM. Texto Contexto Enferm. 2006 Jul-Set;15(3):464-71.

22. Conselho Federal de Enfermagem. Resolução n 358/2009 [página na Internet]. Rio de Janeiro: COFEN; 2011 [cited 2011 May 15]. Available from: www.cofen.br.

23. Possari JF. Centro Cirúrgico: Planejamento, Organização e Gestão. 4. ed. São Paulo: Iátria; 2009.

24. Bocchi SCM, Meneguin S, Santi RC. Sistematização da assistência de enfermagem a paciente com luxação de coluna cervical: estudo de caso. Rev Latino-am Enferm. 1996;4(2):113-29. http://dx.doi. org/10.1590/S0104-11691996000200009

25. Galvão CM, Sawada NO, Trevizan MA. Revisão sistemática: recurso que proporciona a incorporação das evidências na prática da enfermagem. Rev Latino-Am Enferm. 2004 maio/jun;12(3):54956. http://dx.doi.org/10.1590/S0104-11692004000300014 
Apêndice

\section{FACULDADE ASCES \\ PROTOCOLO DE CONSULTA DE ENFERMAGEM - PROJETO DE EXTENSÃO (ENFERMAGEM/ODONTOLOGIA)}

PRÉ-OPERATÓRIO MEDIATO

DADOS PESSOAIS N. do Prontuário:

Nome:

Proposta Cirúrgica:

Sexo: ( ) Masculino ( ) Feminino Idade:

anos

Estado Civil: ( ) Casado ( ) Solteiro ( ) Viúvo ( ) Divorciado

Endereço:

Bairro:

Cidade:

Telefone: ( )

Escolaridade:

Profissão:

Acompanhante:

PADRÃO DE PERCEPÇÃO - MANUTENÇÃO DA SAÚDE

História de Adoecimento anterior: ( ) Sim* ( ) Não. * Especificar:

Grupo sanguíneo: ___ Fator Rh: ( ) Problemas de coagulação: ( ) Sim* ( ) Não. * Especificar:

Faz Uso de medicação: ( ) Sim* ( ) Não. * Especificar:

Alergias: ( ) Sim* ( ) Não. *Especificar:

Fumante: ( ) Sim ( ) Não/Uso de Álcool: ( ) Sim ( ) Não/Realiza atividades físicas: ( )Sim ( ) Não

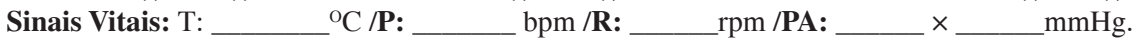

\section{PADRÃO PERCEPTIVO - COGNITIVO}

Refere dor: ( ) Sim* ( ) Não. *Especificar:

Fala: ( ) Normal ( ) Alterada

Nível de consciência: ( ) Consciente ( ) Confuso ( ) Inconsciente

Nível de compreensão: ( ) Normal ( ) Alterado

Nível de orientação: ( ) Orientado ( ) Desorientado*. *Especificar: ( ) No tempo ( ) No espaço ( ) Em pessoas

Visão: ( ) Normal ( ) Diminuída ( ) Ausente ( ) Faz uso de óculos / Audição: ( ) Normal ( ) Diminuída

\section{INTEGRIDADE DO EGO}

Cirurgia prévia: ( ) Sim ( ) Não. Procedimento anestésico anterior: ( ) Sim* ( ) Não. Especificar:

Tem conhecimento sobre o procedimento que será realizado: ( ) Sim ( ) Não

Foram dadas informações a respeito do procedimento: ( ) Sim ( ) Não

\section{AVALIAÇÃO CARDIORRESPIRATÓRIA}

Ausculta cardíaca:

Ausculta pulmonar:

\section{PADRÃO NUTRICIONAL METABÓLICO}

Ingesta hídrica: ( ) Sim ( ) Não. / Apresenta dificuldades para deglutir: ( ) Sim ( ) Não Refluxo após alimentar-se: ( ) Sim ( ) Não Apresenta dificuldades para mastigar: ( ) Sim ( ) Não. / Tem preferência por alimentos quentes: ( ) Sim ( ) Não

\section{DIAGNÓSTICOS DE ENFERMAGEM}

( ) Conhecimento Deficiente. ( ) Medo. ( ) Ansiedade. ( ) Risco de Infecção.

Outros* - *Especificar:

\section{PRESCRIÇÕES DE ENFERMAGEM}

Fornecer informações a respeito da higiene ( )

Fornecer informações a respeito do processo cirúrgico-anestésico ( )

Fornecer informações a respeito da necessidade de acompanhamento: ( ) Médico ( ) Nutricional

Fornecer informações a respeito da necessidade de autocuidado para alta ambulatorial ( )

Outras prescrições: 
Apêndice

\begin{tabular}{|c|c|}
\hline \multicolumn{2}{|l|}{ Data: $\_\_l l \_$Rubrica do Acadêmico: } \\
\hline \multirow{4}{*}{ 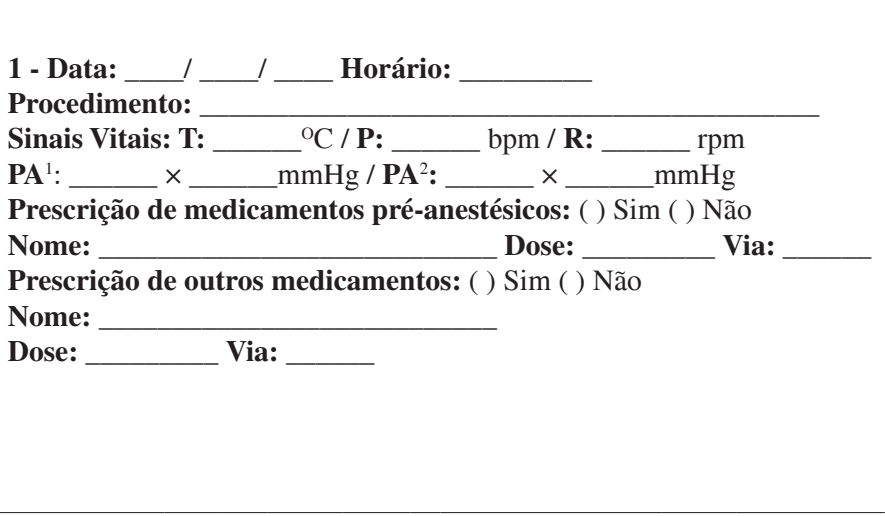 } & DIAGNÓSTICOS DE ENFERMAGEM \\
\hline & $\begin{array}{l}\text { ( ) Risco de trauma ( ) Risco de infecção ( ) Medo } \\
\text { ( ) Ansiedade ( ) Risco de temperatura corporal diminuída } \\
\text { ( ) Outros: }\end{array}$ \\
\hline & PRESCRIÇÕES DE ENFERMAGEM \\
\hline & $\begin{array}{l}\text { ( ) Explicar os procedimentos cirúrgico-anestésicos ao cliente } \\
\text { ( ) Orientar cliente e acompanhante como evitar infecções } \\
\text { ( ) Utilizar técnicas de distração e relaxamento } \\
\text { ( ) Acompanhar o paciente até o equipo de procedimento } \\
\text { ( ) Aquecer o paciente com cobertor no transoperatório } \\
\text { ( ) Verificar e orientar higiene oral } \\
\text { ( ) Orientar técnicas de controle da respiração }\end{array}$ \\
\hline \multirow{4}{*}{ 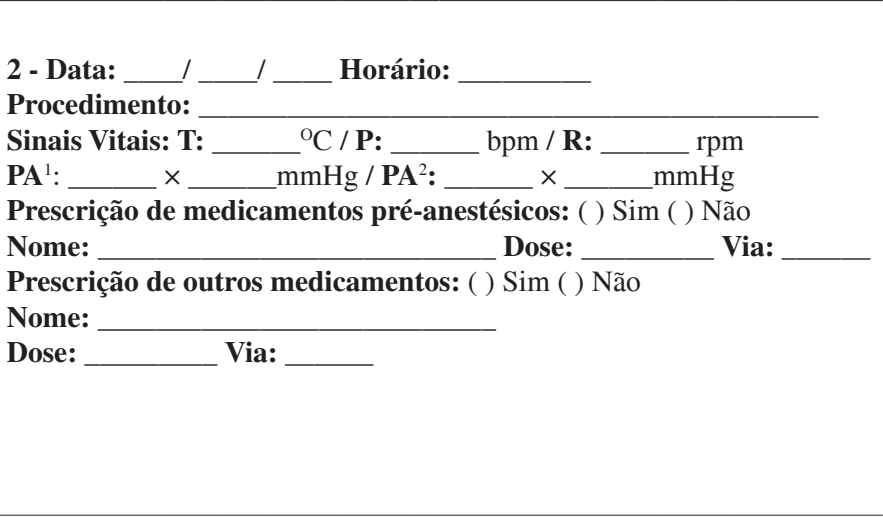 } & DIAGNÓSTICOS DE ENFERMAGEM \\
\hline & $\begin{array}{l}\text { ( ) Risco de trauma ( ) Risco de infecção ( ) Medo } \\
\text { ( ) Ansiedade ( ) Risco de temperatura corporal diminuída } \\
\text { ( ) Outros: }\end{array}$ \\
\hline & PRESCRIÇÕES DE ENFERMAGEM \\
\hline & $\begin{array}{l}\text { ( ) Explicar os procedimentos cirúrgico-anestésicos ao cliente } \\
\text { ( ) Orientar cliente e acompanhante como evitar infecções } \\
\text { ( ) Utilizar técnicas de distração e relaxamento } \\
\text { ( ) Acompanhar o paciente até o equipo de procedimento } \\
\text { () Aquecer o paciente com cobertor no transoperatório } \\
\text { ( ) Verificar e orientar higiene oral } \\
\text { ( ) Orientar técnicas de controle da respiração }\end{array}$ \\
\hline \multirow{4}{*}{ 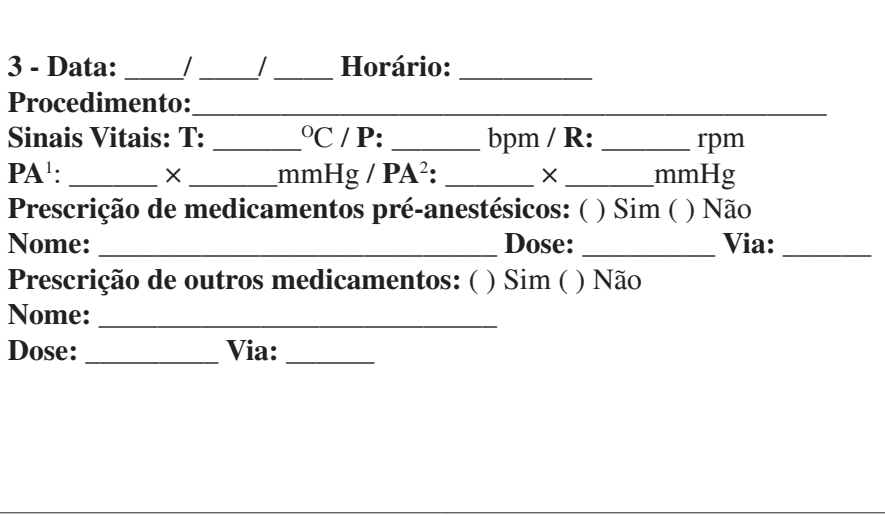 } & DIAGNÓSTICOS DE ENFERMAGEM \\
\hline & $\begin{array}{l}\text { ( ) Risco de trauma ( ) Risco de infecção ( ) Medo } \\
\text { ( ) Ansiedade ( ) Risco de temperatura corporal diminuída } \\
\text { ( ) Outros: }\end{array}$ \\
\hline & PRESCRIÇÕES DE ENFERMAGEM \\
\hline & $\begin{array}{l}\text { ( ) Explicar os procedimentos cirúrgico-anestésicos ao cliente } \\
\text { ( ) Orientar cliente e acompanhante como evitar infecções } \\
\text { ( ) Utilizar técnicas de distração e relaxamento } \\
\text { ( ) Acompanhar o paciente até o equipo de procedimento } \\
\text { ( ) Aquecer o paciente com cobertor no transoperatório } \\
\text { ( ) Verificar e orientar higiene oral } \\
\text { ( ) Orientar técnicas de controle da respiração }\end{array}$ \\
\hline \multirow{4}{*}{ 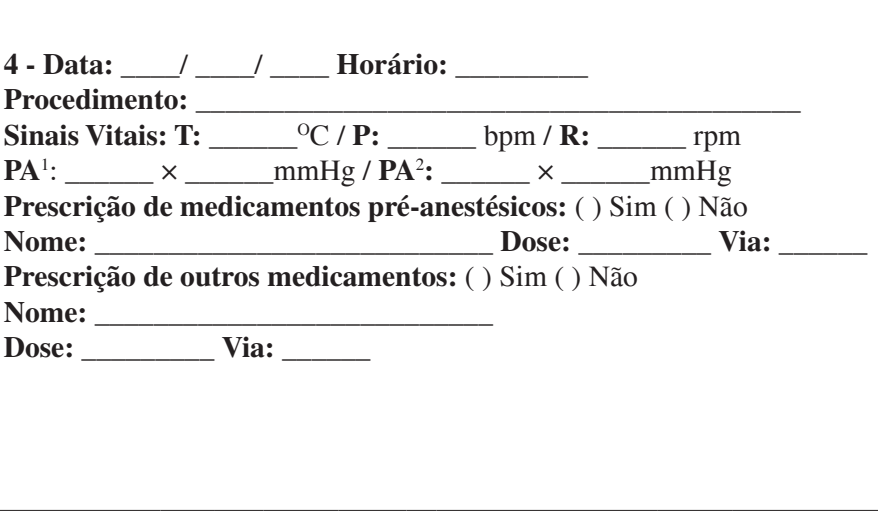 } & DIAGNÓSTICOS DE ENFERMAGEM \\
\hline & $\begin{array}{l}\text { ( ) Risco de trauma ( ) Risco de infecção ( ) Medo } \\
\text { ( ) Ansiedade ( ) Risco de temperatura corporal diminuída } \\
\text { ( ) Outros: }\end{array}$ \\
\hline & PRESCRIÇÕES DE ENFERMAGEM \\
\hline & $\begin{array}{l}\text { ( ) Explicar os procedimentos cirúrgico-anestésicos ao cliente } \\
\text { ( ) Orientar cliente e acompanhante como evitar infecções } \\
\text { ( ) Utilizar técnicas de distração e relaxamento } \\
\text { ( ) Acompanhar o paciente até o equipo de procedimento } \\
\text { ( ) Aquecer o paciente com cobertor no transoperatório } \\
\text { ( ) Verificar e orientar higiene oral } \\
\text { ( ) Orientar técnicas de controle da respiração }\end{array}$ \\
\hline
\end{tabular}


Apêndice

Demonstra conhecimento sobre o procedimento cirúrgico: ( ) Sim ( ) Não

Tosse: ( ) Sim ( ) Não / Jejum: ( ) Sim ( ) Não

Condições de higiene na área do procedimento: ( ) Satisfatória ( ) insatisfatória

Hora da última refeição: ___ h. / Uso de medicação: ( ) Sim ( ) Não

Tipo da medicação: Especificar:

Resultados Laboratoriais normais: ( ) Sim ( ) Não* - *Especificar:

\section{TRANSOPERATÓRIO}

1 - Data: / / Horário:

Procedimento:

Anestésico utilizado: Outros:

Intercorrências cirúrgicas: ( ) Sim* ( )

Não

Intercorrências anestésicas: ( ) Sim* ( ) Não *Quais:

Extensionista:

Aluno/cirurgião responsável:

SSVV: PA: __ $\times \_\mathrm{mmHg} / \mathbf{P}: \_$bpm

2 - Data: / / Horário: Procedimento:

Anestésico utilizado: ___ Outros:

Intercorrências cirúrgicas: ( ) Sim* ( )

Não

Intercorrências anestésicas: ( ) Sim* ( ) Não *Quais:

Extensionista:

Aluno/cirurgião responsável:

SSVV: PA:

$\mathrm{mmHg} / \mathbf{P}$

bpm

3 - Data: ___ ___ Horário:____ Procedimento:

Anestésico utilizado: ___ Outros: Intercorrências cirúrgicas: ( ) Sim* ( )

Não

Intercorrências anestésicas: ( ) Sim* ( ) Não *Quais:

Extensionista:

Aluno/cirurgião responsável:

SSVV: PA: $\times$

$\mathrm{mmHg} / \mathbf{P}:$ bpm

4 - Data: ___ ___ Horário:___ Procedimento:

Anestésico utilizado:

Outros:

Intercorrências cirúrgicas: ( ) Sim* ( )

Não

Intercorrências anestésicas: ( ) Sim* ( ) Não *Quais:

Extensionista:

Aluno/cirurgião responsável:

SSVV: PA: _ _ _ $\mathrm{mmHg} / \mathbf{P :} \_$_ $b p m$

\section{OPERATÓRIO IMEDIATO/MEDIATO}

\begin{tabular}{|c|c|c|c|c|c|}
\hline DATA & HORÁRIO & $\mathbf{T}$ & $\mathbf{R}$ & $\mathbf{P}$ & PA \\
\hline \multirow[t]{2}{*}{11} & & ${ }^{\circ} \mathrm{C}$ & $\mathrm{rpm}$ & bpm & ${ }_{\mathrm{mmHg}}^{\times}$ \\
\hline & & ${ }^{\circ} \mathrm{C}$ & $\mathrm{rpm}$ & bpm & ${ }_{\mathrm{mmHg}}^{\times}$ \\
\hline \multirow[t]{2}{*}{1} & & ${ }^{\circ} \mathrm{C}$ & $\mathrm{rpm}$ & bpm & $\underset{\mathrm{mmHg}}{\times}$ \\
\hline & & ${ }^{\circ} \mathrm{C}$ & $\mathrm{rpm}$ & bpm & ${ }^{\times} \mathrm{mmHg}$ \\
\hline
\end{tabular}

PÓS-OPERATÓRIO MEDIATO/PROSERVAÇÃO

\begin{tabular}{|c|c|c|c|c|}
\hline SSVV & DATA $^{1}: \ldots$ & DATA $^{2}: \ldots$ & DATA $^{3}: \ldots 1$ & $\begin{array}{c}\text { DATA }^{4}: \\
1 / 1 \\
\end{array}$ \\
\hline \multirow[t]{2}{*}{ Pressão Arterial } & ${ } \times \ldots \mathrm{mmHg}$ & $\ldots$ m $\mathrm{mmHg}$ & 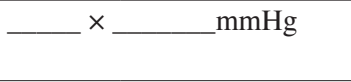 & $\stackrel{\mathrm{mmHg}}{\mathrm{m}}$ \\
\hline & ${ } \times \ldots \mathrm{mmHg}$ & $\ldots \mathrm{mmHg}$ & ${ } \times \ldots \mathrm{mmHg}$ & $\stackrel{\mathrm{mmHg}}{\mathrm{m}}$ \\
\hline \multirow[t]{2}{*}{ Pulso } & bpm & bpm & bpm & bpm \\
\hline & bpm & bpm & bpm & bpm \\
\hline
\end{tabular}


Apêndice

\section{DIAGNÓSTICOS E PRESCRIÇÕES DE ENFERMAGEM PARA ALTA DA SRPA}

Checar códigos correspondentes: (1) DATA ${ }^{1}: \ldots / \ldots$ 1

$(1,2,3,4)$ Comunicação $\quad(1,2,3,4)$ Investigar a capacidade de compreender e falar.

prejudicada

$(1,2,3,4)$ Proporcionar métodos alternativos de comunicação.

$(1,2,3,4)$ Comprovar que a mensagem foi compreendida.

$(1,2,3,4)$ Outras:

$(1,2,3,4)$ Conforto

$(1,2,3,4)$ Explicar fatores que agravam sintomas.

prejudicado

$(1,2,3,4)$ Promover o conforto e prevenir lesão mais profunda.

$(1,2,3,4)$ Outras:

$(1,2,3,4)$ Dor aguda

$(1,2,3,4)$ Administrar medicamentos prescritos.

$(1,2,3,4)$ Reconhecer a presença da dor, bem como ouvir atentamente o que é dito em relação à dor.

$(1,2,3,4)$ Outras:

$(1,2,3,4)$ Integridade da

pele prejudicada

$(1,2,3,4)$ Instruir a comunicar o desconforto.

$(1,2,3,4)$ Aplicar compressa fria no local cirurgiado (EXTERNA).

$(1,2,3,4)$ Outras:

$(1,2,3,4)$ Padrão

respiratório ineficaz

$(1,2,3,4)$ Posicionar em Fowler ou semifowler.

$(1,2,3,4)$ Instalar oxigenoterapia.

$(1,2,3,4)$ Ensinar técnicas de respiração controlada com o profissional.

$(1,2,3,4)$ Garantir que estão sendo tomadas medidas para manter sua segurança.

$(1,2,3,4)$ Outras:

\section{ORIENTAÇÕES GERAIS}

\begin{tabular}{|c|c|c|c|c|}
\hline DATA $^{1}:$ & DATA $^{2}:$ & DATA $^{3}:$ & $\begin{array}{c}\text { DATA }^{4}: \\
1 \\
\end{array}$ & ORIENTAÇÕES GERAIS \\
\hline ( ) & () & ( ) & () & $\begin{array}{l}\text { Decúbito: a cabeça deve estar virada para o lado oposto ao da } \\
\text { cirurgia. }\end{array}$ \\
\hline () & () & () & () & $\begin{array}{l}\text { Repouso: manter o repouso nas primeiras } 24 \text { horas para evitar } \\
\text { sangramentos e edema. }\end{array}$ \\
\hline ( ) & ( ) & ( ) & ( ) & $\begin{array}{l}\text { Medicação: seguir corretamente as prescrições; caso apareça } \\
\text { reação alérgica a alguma medicação, esta deve ser suspensa e o } \\
\text { odontologista responsável deve ser comunicado. }\end{array}$ \\
\hline ( ) & ( ) & ( ) & ( ) & Não praticar automedicação. \\
\hline ( ) & () & ( ) & () & $\begin{array}{l}\text { Edema: utilizar compressa de gelo na região (parte externa) } \\
\text { alternando a cada } 15 \text { min. nas primeiras } 48 \mathrm{~h} \text {. }\end{array}$ \\
\hline ( ) & ( ) & ( ) & ( ) & $\begin{array}{l}\text { Bochecho: evitar bochecho nas primeiras horas para não } \\
\text { remover o coágulo (tampão hemostático) e, quando for prescrito } \\
\text { o bochecho, este deve ser realizado suavemente. }\end{array}$ \\
\hline () & () & ( ) & () & $\begin{array}{l}\text { Escovação: deve ser realizada normalmente, desde que não } \\
\text { traumatize a área lesionada. }\end{array}$ \\
\hline ( ) & $($ ) & $($ ) & ( ) & $\begin{array}{l}\text { Hemorragia: pequenas quantidades de sangue são esperadas, } \\
\text { mas sangramentos abundantes devem ser informados ao } \\
\text { cirurgião-dentista. }\end{array}$ \\
\hline ( ) & $($ ) & ( ) & ( ) & $\begin{array}{l}\text { Dieta: evitar alimentos quentes, dando preferência a alimentos } \\
\text { líquidos e pastosos, com temperatura fria ou gelada. }\end{array}$ \\
\hline
\end{tabular}

ANOTAÇÕES: 
Apêndice

\begin{tabular}{|l|l|l|l|}
\hline \multicolumn{2}{|l|}{ EVOLUÇÃO DE ENFERMAGEM } & RUBRICA \\
\hline DATA & HORÁRIO & EVOLUÇÃO & \\
\hline & & & \\
\hline & & & \\
\hline & & & \\
\hline
\end{tabular}

\section{RASTREABILIDADE!}

Um dos tópicos mais importantes da RDC 15/2012.

Sua incubadora fornece resultados impressos? Libere sua carga de implantes com segurança e documentação.

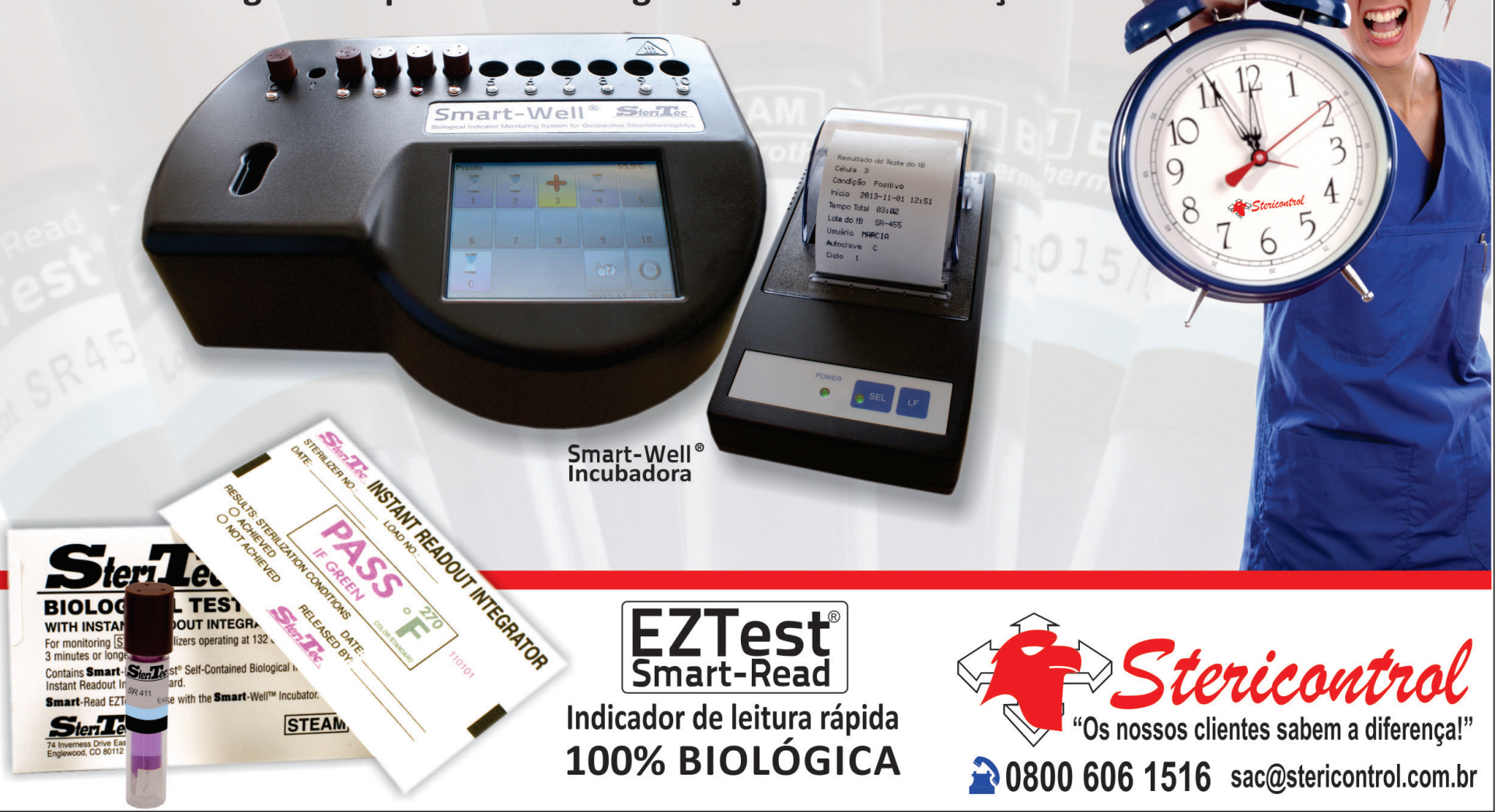

